\title{
Cytoplasmic APE1 promotes lung cancer aggressiveness and cisplatin resistance via the COX-2/Akt/ $\beta$-catenin signaling pathway
}

\section{Zhimin Zhang}

Third Military Medical University Daping Hospital and Research Institute of Surgery

\section{Xiaojuan Lian}

Jiangjin Strict hospital

\section{Xiaona Su}

Third Military Medical University Daping Hospital and Research Institute of Surgery

\section{Maojun Liao}

Third Military Medical University Daping Hospital and Research Institute of Surgery

\section{Chuan Chen}

Third Military Medical University Daping Hospital and Research Institute of Surgery

\section{Ge Wang ( 2065397459@qq.com )}

Third Military Medical University Daping Hospital and Research Institute of Surgery

\section{Research}

Keywords: Cytoplasmic APE1, COX-2, Overall survival, Lung cancer, Cisplatin resistance

Posted Date: September 23rd, 2020

DOl: https://doi.org/10.21203/rs.3.rs-78089/v1

License: (c) (1) This work is licensed under a Creative Commons Attribution 4.0 International License. Read Full License 


\section{Abstract \\ BACKGROUND}

Cisplatin is commonly used in lung cancer therapy, but cisplatin resistance in lung cancer cells remains an unsolved problem. Here, we report that cytoplasmic APE1 contributes to cisplatin resistance, cell proliferation and migration in lung cancer cells.

\section{METHODS}

Immunofluorescence, western blot analysis, lentivirus transfection and scratch assays, and transwell migration and invasion assays were carried out on the cell lines A549 and Calu-1. A total of 124 samples of lung cancer tissues were evaluated to determine the clinical effects of cytoplasmic APE1 and COX-2.

\section{RESULTS}

We found that cytoplasmic APE1 expression was lower in cisplatin sensitive cells than in cisplatinresistant cells, and the upregulation of cytoplasmic APE1 significantly reduced cisplatin sensitivity in lung cancer cells. Gain-of-function studies demonstrated that cytoplasmic APE1 promoted lung cancer cell proliferation, migration and invasion in vitro and tumor growth in vivo, which were inhibited after cisplatin treatment. In patient samples, cytoplasmic APE1 in lung cancer tissues was an independent indicator of overall survive $(O S)$ for lung cancer patients $(P<0.001)$. Mechanistic studies revealed that cytoplasmic APE1 promotes lung cancer malignancy by activating the COX-2/Akt/ $\beta$-catenin pathway. Furthermore, the mutation of the APE1-C65 site caused upregulation of cytoplasmic APE1 and inhibited cell growth, migration and invasion of lung cancer cells.

\section{CONCLUSION}

We suggest that modulating cytoplasmic APE1 in lung cancer is a promising novel strategy for overcoming cisplatin resistance.

\section{Background}

Lung cancer is the most common cancer in the world, and cisplatin-based chemotherapy is frequently used in clinical treatments. However, resistance to cisplatin is common during the treatment of lung cancer, leading to low overall survival (OS) and a low objective response rate (ORR) to cisplatin in lung cancer. Therefore, further investigation into the mechanism of cisplatin resistance is urgently warranted.

Apurinic/apyrimidinic endonuclease 1/redox factor-1 (APE1/Ref-1) is a multifunctional enzyme that acts as a key AP endonuclease during base excision repair. APE1 redox activity maintains the activity of 
transcription factors [1, 2]. APE1 contains a subcellular localization signal; the amino-acid sequence of human APE1 has an identified consensus nuclear localization signal (NLS) within its N-terminus, which has been confirmed to function in subcellular targeting [3]. The current data suggest a model in which unmodified, full-length APE1 is mainly targeted to the nucleus via its $\mathrm{N}$-terminal elements [3]. More importantly, APE1 is widely overexpressed in most tumor tissues, and APE1 overexpression is associated with poor prognosis, chemoresistance or radioresistance, and increased angiogenesis [4-6]. In this study, cytoplasmic APE1 may mediate resistance to cisplatin in lung cancer, and upregulation of cytoplasmic APE1 induced by plasmid transfection increased resistance to cisplatin.

Here, we propose that cytoplasmic APE1 causes COX-2 mediated cisplatin resistance in lung cancer and that cytoplasmic APE1 expression is significantly higher in lung cancer patients who have poor OS after cisplatin-based chemotherapy. Cytoplasmic APE1 regulates cancer traits in lung cancer cells, including proliferation, metastasis and invasion. In addition, cytoplasmic APE1 overexpression activates the Akt/ $\beta$ catenin signaling pathway by upregulating COX-2 in lung cancer cells.

\section{Materials And Methods}

\section{Materials}

Cisplatin (cDDP), penicillin were from Sigma-Aldrich (USA). Antibodies against APE1, COX-2 and Histone 1 were from Santa Cruz Biotechnology (USA). Antibodies against $\beta$-tubulin, P-Akt, and Akt were from Abcam (USA). The CCK-8 assay, RIPA assay, Transwell $®$ chambers and crystal violet were from Beyotime Corporation (China).

\section{Cell culture}

Calu-1 and A549 cell lines were from the American Type Culture Collection (ATCC, USA). The cDDPresistant A549 (A549-cDDP) cell line was kindly provided by Xinqiao Hospital, Army Medical University. A549-cDDP cells screening procedure: A549 cells growing in logarithmic phase was firstly treated with a concentration of $0.1 \mu \mathrm{M}$ cisplatin for 48 hours. The cells were subsequently incubated with normal medium until reached to logarithmic phase again. This process was repeatedly conducted with increasing cisplatin concentration by $0.1 \mu \mathrm{M}$ very cycle. Finally, these cells grew well in cisplatin at a concentration of $4 \mu \mathrm{M}$. Calu- 1 and A549 cells were cultured in DMEM medium containing $10 \%$ FBS and $37^{\circ} \mathrm{C}$ humidified incubator with $5 \% \mathrm{CO} 2$.

\section{Transfection}

shRNA-APE1 (shAPE1: the 33 amino-acid N-terminal truncated form, NLS mutation of APE1) and shRNAcon (shCON, the full-length form) plasmid[7], shRNA-C65s (shC65S: C65 site mutation of APE1) and shRNA-con (shCON) plasmid [8], and siRNA-COX-2 (siCOX-2) and siRNA-con (siCON) plasmid were purchased from Genechem Corporation (Shanghai, China). Using the Silencer siRNA construction kit (Qiagen, Venlo, The Netherlands), the cells were performed with the RNAiFect reagents (The Netherlands) to ensure the stability of small RNAi transfection [9]. 


\section{Western blot analysis}

Equal amounts of proteins were separated on gels, then transferred to membranes. The membranes were blocked, incubated with the primary antibody, and then incubated secondary antibodies. Protein bands were visualized, the protein bands were analyzed (Bio-Rad, USA).

\section{CCK-8 assay}

Cells were seeded and treated with the indicated conditions. Each well was added 10 ul CCK-8 reagent and was incubated for 2 hour. And then Microplate Reader 550 (Bio-Rad) determined the optical density (OD). The inhibitory effect was calculated through dividing OD of samples underwent various treatments by OD of controls after subtracting OD of blanks.

\section{Scratch assay and transwell assay}

Cells were seeded and treated with the indicated conditions. Cell monolayers were scratched and removed detached cells. Olympus microscope imaged the wounded gap.

Cells were seeded into the upper chamber with 8- $\mu \mathrm{m}$ pore size transwell filters, In the lower chamber, the DMEM's minimal essential medium was place as attractant. After $18 \mathrm{~h}$ of incubation, the filters was stained, cells of filters were observed by bright field microscopy.

\section{In vivo experiment}

Female BALB/C nude mice were from the Daping Hospital (China), the mice were injected A549 cells that were stably transfected with either a plasmid containing APE1 having a NSL mutation (shAPE1) or the vehicle (shCON). Cisplatin (CDDP) was delivered via intraperitoneal injection for 3 weeks. Measuring tumor diameter and calculating tumor volume was as follows: $a b 2 / 2 \mathrm{~mm}^{3}$ ( $a=$ length; $b=$ width), where is the length and is the width of the tumor. Mice were randomized: shCON (PBS), shAPE1, and a combination of shAPE1 + cDDP [10].

\section{Immunofluorescence (IF) and Immunohistochemistry (IHC) analysis}

For IF analysis, collected cell were permeabilized, blocked, and incubated with the first antibody. Then, the cells were flooded and stained with DAPI. Zeiss microscope performed transmission electron microscopy analysis (Carl Zeiss $\mathrm{GmbH}$, Oberkochen, Germany). Images were captured using a confocal microscope (40 $\times$ magnification), data are observed from more than three microscopic fields of view per.

For IHC analysis, the slides were baked for 1 hour at $60^{\circ} \mathrm{C}$, soaked with xylene for 30 minutes, soaked in different concentrations of alcohol for 5 seconds and were immersed in a pressure cooker containing citric acid buffer and heated to air jet for 2 minutes. The slides were incubated with PARP1 and p62 monoclonal antibody (1:100 and 1:50 dilution), and then with secondary antibody (1:50 dilution). 
Cytoplasmic PARP1 and p62 staining score was performed. Weak staining and $<50 \%$ of stained cells were defined as low expression [11].

\section{Patients}

We selected 124 patients with unresectable, locally advanced or metastatic lung cancer (table S1); the enrolled patients were treated between July 2006 and July 2015 at the Daping Hospital of the Army Medical University of China. Tissue samples of lung cancer patients before cisplatin basedchemotherapy were collected, and were evaluated the clinic effect of cytoplasmic APE1 and COX-2.

\section{Statistical analysis}

Kaplan-Meier method analyzed the OS and PFS, statistical analysis was analyzed using SPSS 20.0.0 (USA). Two-sided P-values $<0.05$ were considered significant.

\section{Results}

\section{Overexpression of cytoplasmic APE1 induced cisplatin resistance in lung cancer}

To explore the mechanism of cisplatin resistance, we constructed and identified cisplatin resistant A549 cells (A549-cDDP) and cisplatin sensitive A549 cells (A549-CON) (Fig. 1A). We found that both cytoplasmic and total APE1 expression levels were higher in the cisplatin-resistant cell line A549-cDDP than in A549-CON (Fig. 1B). These results were further demonstrated in more detail by immunofluorescence (Fig. 1C). It was clearly observed that the upregulation of APE1 expression was restricted to the cytoplasm rather than the nuclear area (Figure S1A). The relationship between APE1 expression and cisplatin resistance has been widely confirmed [12]. We believe that cytoplasmic APE1 may play an important role in the mechanism of resistance to cisplatin. These results were further confirmed in another lung cancer cell line Calu-1 by overexpressing cytoplasmic APE1 through transfection of a plasmid expressing APE1 with an NLS inactive mutation. As shown in Fig. 1D and Figure S1B, cisplatin treatment increased cytoplasmic APE1 levels and total APE1 levels in Calu-1 cells in a similar manner to that observed in A549 cells. Overexpression of cytoplasmic APE1 significantly increased the cisplatin resistance and proliferation of Calu-1 cells (Fig. 1E and F) after cisplatin treatment. These results indicated that the upregulation of cytoplasmic APE1 expression after cisplatin treatment was more pronounced in A549-CON cells than in A549-cDDP cells. Therefore, we proposed that cytoplasmic APE1 could be the major cause of cisplatin resistance.

\section{Effects of cytoplasmic APE1 on cell proliferation, migration and invasion}

Then, we investigated the role of cytoplasmic APE1 in cisplatin sensitivity. Overexpression of cytoplasmic APE1 in A549 and Calu-1 cells (Fig. 2A) substantially increased cell growth, cell migration and invasive 
capacity following cisplatin treatment (Figure. 2B-D). The cell growth, migration and invasion capacities were higher in the control group than in the CDDP alone group, and the level of cytoplasmic APE1 in the cDDP alone group was higher than that in the control group and lower than that in the group treated with shAPE1 and cisplatin, as shown in Figure. 1 and Figure. 2, so the cDDP alone group is not displayed. These results suggested that cytoplasmic APE1 promoted the growth, migration and invasion of lung cancer cells after cisplatin treatment.

\section{Overexpression of cytoplasmic APE1 promoted tumor growth in vivo}

We also researched the impact of cytoplasmic APE1 on cisplatin resistance in vivo by using xenograft tumors in nude mice consisting of A549 cells expressing either a control (shCON) or cytoplasmic APE1overexpression vector (shAPE1). We observed that overexpression of cytoplasmic APE1 significantly increased tumor growth and resistance to cisplatin in vivo (Figure. 3A-B). Western blot results in xenograft tumors also revealed that NLS inactive mutated APE1 (shAPE1) increased cytoplasmic APE1 expression and that cisplatin induced the expression of cytoplasmic and total APE1 in vivo (Fig. 3C). These results were further verified by immunohistochemical analysis (Fig. 3D). Cisplatin induced the expression of cytoplasmic and total APE1, and cytoplasmic expression enhanced resistance to cisplatin in vivo.

\section{The effect of cytoplasmic APE1 was affected by COX-2 levels}

Our further studies showed that the overexpression of cytoplasmic APE1 significantly increased COX-2 expression in lung cancer cells (Fig. 4A). These results were verified by immunohistochemistry staining of COX-2 expression in xenograft tumors, and both cisplatin and cytoplasmic APE1 induced the expression of COX-2 (Fig. 4B). To explore whether COX-2 is a functional target of cytoplasmic APE1, A549 cells were transfected with shRNA plasmids against COX-2 (siCOX-2). COX-2 expression was induced in siCON cells but remained depressed in siCOX-2 cells under CDDP treatment (Fig. 4C). As expected, cytoplasmic APE1 overexpression attenuated the inhibition of cell proliferation, migration, and invasion conferred by cisplatin treatment, and the downregulation of COX-2 attenuated the enhancement in cell proliferation, migration, and invasion conferred by cytoplasmic APE1 overexpression (Fig. 4D-F). The downregulation of COX-2 directly regulated cell proliferation, migration, and invasion associated with cisplatin treatment and cytoplasmic APE1 expression. These data revealed that COX-2 expression plays an important role in mediating the effects of cytoplasmic APE1.

\section{Cytoplasmic APE1 promoted the Akt/ $\beta$-catenin signaling pathway by targeting COX-2 in lung cancer}

As previously shown, COX-2 was located upstream of the Akt/ $\beta$-catenin signaling pathway $([13,14]$ and Fig. 4C). Accordingly, we sought to use cytoplasmic APE1 to probe the role of COX-2 in the Akt/ $\beta$-catenin 
signaling in A549 cells. We demonstrated that overexpression of cytoplasmic APE1 increased $\beta$-catenin and phosphorylated Akt, but cisplatin treatment reduced $\beta$-catenin and phosphorylated Akt (Fig. 5A). As expected, both COX-2 inhibition and cisplatin treatment decreased the levels of $\beta$-catenin and phosphorylated Akt (Fig. 5B). Furthermore, western blot results further verified that cisplatin inhibited the activation of the Akt/ $\beta$-catenin signaling pathway conferred by the overexpression of cytoplasmic APE1 in vivo (Fig. $5 \mathrm{C}$ ). Overall, overexpression of cytoplasmic APE1 activated the COX-2/Akt/ $\beta$-catenin signaling pathway, and cisplatin increased cytoplasmic APE1 but inhibited the COX-2/Akt/ $\beta$-catenin signaling pathway. Therefore, we believe that cytoplasmic APE1 promotes cell proliferation and cisplatin resistance by activating the COX-2/Akt/ $\beta$-catenin signaling pathway, reversing its inhibition by cisplatin.

\section{Clinical prognostic values of cytoplasmic APE1 and COX-2}

To evaluate the clinical value of cytoplasmic APE1 and COX-2 after cisplatin based chemotherapy in lung cancer patents, we analyzed cytoplasmic APE1 and COX-2 expression in 124 tissue blocks obtained from biopsies of lung cancer tissues by immunohistochemistry (table S1). Immunohistochemistry analyses showed that APE1 staining was localized in the cytoplasm and nucleus, while COX-2 was mostly localized to the cytoplasm. The intensity and percentage of cytoplasmic APE1 and COX-2 staining were determined for each of the clinical cases using a protocol previously described by our laboratory, as well as other investigators. Kaplan-Meier curves showed that high levels of cytoplasmic APE1 in the tumor sample significantly correlated with shorter OS (median time to progression of low-expression vs highexpression of cytoplasmic APE1: 19.0 months vs. 8.0, respectively, $\mathrm{P}<0.005$; Kaplan-Meier curves are shown in Fig. 5D) and that the COX-2 high-expression group had a shorter OS than the low-expression group (median time to progression of low-expression vs high-expression of COX-2: 14.0 months vs. 12.0, respectively, $\mathrm{P}<0.005$; Kaplan-Meier curves shown in Fig. 5E). These data revealed that both cytoplasmic APE1 and COX-2 are independent prognostic indicators in patients with lung cancer (Table S2).

\section{Cytoplasmic APE1 was downregulated by mutation of APE1-C65 site}

Finally, we explored the molecular mechanisms responsible for cytoplasmic APE1 in lung cancer cells. The APE1-C65 site plays an important role in the redox function of APE1, and we next examined whether APE1-C65 mutations can impair cytoplasmic APE1 expression and therefore recover cisplatin sensitivity. We investigated the role of cytoplasmic APE1 in cisplatin sensitivity using a plasmid expressing APE1 with an APE1 C65 site inactive mutation (shC65S). shC65S induced cytoplasmic APE1 expression in A549 cells (Fig. 6A), which significantly reduced resistance to cisplatin, cell growth, cell migration and invasion ability. Moreover, the reduced cell growth, migration and invasion induced by shC65S were further suppressed after cisplatin treatment (Fig. 6B-D). Phosphorylated Akt and $\beta$-catenin downstream of the COX-2/Akt/ $\beta$-catenin signaling pathway were also inhibited by shC65S and shC65S plus cisplatin treatment (Fig. 6E). Taken together, APE1-C65 site mutations could increase cytoplasmic APE1 expression and resistance to cisplatin in lung cancer cells. 


\section{Discussion}

We reported that APE1 overexpression was associated with cisplatin resistance in lung cancer [12]. However, the detailed mechanism of APE1 in cisplatin resistance remains elusive. In this study, we have found that cytoplasmic APE1 can cause resistance to cisplatin by upregulating COX-2 expression in lung cancer. Additionally, cytoplasmic APE1 expression is important in the occurrence of poor prognosis in lung cancer. Thus, we conclude that cytoplasmic APE1 can predict certain prognostic outcomes for lung cancer patients. Specifically, cytoplasmic APE1 expression can be used as a new method of identifying lung cancer patients with high tumor metastasis risk. These findings underscore the role of cytoplasmic APE1 expression in the development and progression of lung cancer.

Several reports have documented the associations between cytoplasmic APE1 expression and lung tumor aggressiveness, patient survival and relapse in lung cancer; additionally, patient prognosis in human epithelial ovarian cancer and anti-inflammatory activity in cultured endothelial cells have also been linked to cytoplasmic APE1 levels [7, 15-17]. In this study, we explored the role of cytoplasmic APE1 in the malignant phenotype of lung cancer in vitro and in vivo. Our results demonstrated that cytoplasmic APE1 can promote lung cancer cell growth and aggressiveness by inactivating the Akt/ $\beta$-catenin signaling pathway by increasing COX-2 levels.

Cytoplasmic APE1 activity could be the major factor in cisplatin resistance induced by the overexpression of APE1. In our results, cell lines with cisplatin resistance had high cytoplasmic APE1 levels, and upregulation of cytoplasmic APE1 in cisplatin-sensitive cells caused resistance to cisplatin. Lower expression of cytoplasmic APE1 in lung cancer tissues was linked to better OS in patient samples. These results are entirely consistent with our earlier research that showed that cisplatin treatment increases APE1 expression and that inhibiting APE1 expression significantly increases the sensitivity of lung cancer cells to cisplatin $[11,18]$. Clinical data analysis showed that survival prognosis was significantly better in lung cancer patients with low APE1 than in those with high APE1 expression levels [11]. The role of APE1 in cisplatin resistance can at least partially attributed to increased cytoplasmic APE1 expression.

The activated Akt/ $\beta$-catenin signaling pathway plays an important role in enhancing the malignant phenotype of human cancer $[19,20]$. It has been reported that COX-2 indirectly regulates the Akt/ $\beta$-catenin signaling pathway, while activated Akt/ $\beta$-catenin signaling is strongly involved in cancer cisplatin resistance [21,22]. The associations between the inhibition of Cox-2 expression, COX-2 inhibitors and cisplatin therapy resistance have been universally confirmed. Akt/ $\beta$-catenin is one of the downstream signaling molecules activated by COX-2 in lung cancer malignancy that is mediated by cytoplasmic APE1.

The main purpose of our study was to explore the role of cytoplasmic APE1 in cisplatin resistance. Two critical questions were raised. First, cytoplasmic APE1 may have two functions: overexpression of cytoplasmic APE1 activated the COX-2/Akt/ $\beta$-catenin signaling pathway and promoted cell proliferation and invasion, but overexpression of cytoplasmic APE1 induced by cisplatin played another role. Therefore, we believe that cytoplasmic APE1 may not only promote cell proliferation and invasion, but 
also reduce the toxicity of cisplatin by other mechanisms. Second, the mechanism by which cisplatin increased the expression of cytoplasmic of APE1 may be through inducing APE1-C65 site mutations. C65 is located within adjacent strands in the middle of one of the beta sheets within APE1, and the APE1-C65s site mutation causes a significant loss of the redox activity of APE1, which may cause the expression of cytoplasmic APE1. Both the APE1-C65 site mutation and cisplatin increased the expression of cytoplasmic APE1 and inhibited the COX-2/Akt/ $\beta$-catenin signaling pathway. Moreover, cisplatin enhanced APE1-C65 site mutation-mediated inhibition of the COX-2/Akt/ $\beta$-catenin signaling pathway. Previous studies have suggested that mutation of the N-terminus of APE1 induces cytoplasmic APE1 expression, specifically in the case of the $\mathrm{C} 65$ mutation, which is involved in cell proliferation [23]. However, our study confirmed that cytoplasmic APE1 can cause cisplatin resistance by activating the COX-2/Akt/ $\beta$-catenin signaling pathway, and the detailed relationships among cisplatin, APE1-C65 site mutations and cytoplasmic APE1 required further study.

Our report describes cytoplasmic APE1 expression in human lung cancers. Increased expression of cytoplasmic APE1 may be important in tumor aggressiveness, poor prognostic phenotype and the acquisition of cisplatin resistance in lung cancer. In addition, overexpression of cytoplasmic APE1 promoted lung cancer cells aggressiveness by the COX-2 downstream signaling pathway. Thus, cytoplasmic APE1 could be a new prognostic marker and target for cisplatin resistance.

\section{Declarations}

\section{Ethics approval and consent to participate:}

Ethics approval was granted by the Ethics Committee of The Army Medical University. Animal experiments were approved according to the Institutional Animal Care and Use Committee of The Army Medical University, and were performed according to the guidelines for the care and use of laboratory animals.

\section{Consent for publication:}

All authors have approved the manuscript and agree with submission to Journal of Experimental and Clinical Cancer Research.

\section{Availability of data and material:}

The data used to support the findings of this study are available from the corresponding author upon request.

\section{Competing interests:}

None declared. 


\section{Funding:}

This work was supported by grants from the National Natural Science Foundation of China (NSFC) No. 81802292 to Zhimin Zhang and NSFC No 81572959 to Ge Wang.

\section{Authors' contributions:}

MJ. L. and XN. S. performed the experiments. MJ. L. analyzed the results. XJ. L and ZM. Z. wrote the manuscript.

\section{References}

1. Barbarov Y, Timaner M, Alishekevitz D, Hai T, Yokoyama KK, Shaked Y, Aronheim A. Host JDP2 expression in the bone marrow contributes to metastatic spread. Oncotarget. 2015;6(35):37737-49.

2. Vazakidou ME, Magkouta S, Moschos C, Psallidas I, Pappas A, Psarra K, Kalomenidis I. Temsirolimus targets multiple hallmarks of cancer to impede mesothelioma growth in vivo. Respirology. 2015;20(8):1263-71.

3. Lashinger LM, O'Flanagan CH, Dunlap SM, Rasmussen AJ, Sweeney S, Guo JY, Lodi A, Tiziani S, White E, Hursting SD. Starving cancer from the outside and inside: separate and combined effects of calorie restriction and autophagy inhibition on Ras-driven tumors. Cancer metabolism. 2016;4:18.

4. Wu YC, Tang SJ, Sun GH, Sun KH. CXCR7 mediates TGFbeta1-promoted EMT and tumor-initiating features in lung cancer. Oncogene. 2016;35(16):2123-32.

5. Liu Y, Liu YS, Wu PF, Li Q, Dai WM, Yuan S, Xu ZH, Liu TT, Miao ZW, Fang WG, et al. Brain microvascular endothelium induced-annexin $A 1$ secretion contributes to small cell lung cancer brain metastasis. Int J Biochem Cell Biol. 2015;66:11-9.

6. Gao L, Sun X, Zhang Q, Chen X, Zhao T, Lu L, Zhang J, Hong Y. Histone deacetylase inhibitor trichostatin $\mathrm{A}$ and autophagy inhibitor chloroquine synergistically exert anti-tumor activity in $\mathrm{H}$-ras transformed breast epithelial cells. Mol Med Rep. 2018;17(3):4345-50.

7. Wu HH, Cheng YW, Chang JT, Wu TC, Liu WS, Chen CY, Lee H. Subcellular localization of apurinic endonuclease 1 promotes lung tumor aggressiveness via NF-kappaB activation. Oncogene. 2010;29(30):4330-40.

8. Jiang Y, Guo C, Vasko MR, Kelley MR. Implications of apurinic/apyrimidinic endonuclease in reactive oxygen signaling response after cisplatin treatment of dorsal root ganglion neurons. Cancer research. 2008;68(15):6425-34.

9. Hagan MP, Yacoub A, Dent P. Radiation-induced PARP activation is enhanced through EGFR-ERK signaling. Journal of cellular biochemistry. 2007;101(6):1384-93.

10. Sui H, Shi C, Yan Z, Li H. Combination of erlotinib and a PARP inhibitor inhibits growth of A2780 tumor xenografts due to increased autophagy. Drug Des Devel Ther. 2015;9:3183-90. 
11. Wang D, Xiang DB, Yang XQ, Chen LS, Li MX, Zhong ZY, Zhang YS. APE1 overexpression is associated with cisplatin resistance in non-small cell lung cancer and targeted inhibition of APE1 enhances the activity of cisplatin in A549 cells. Lung cancer. 2009;66(3):298-304.

12. Ren T, Shan J, Li M, Qing Y, Qian C, Wang G, Li Q, Lu G, Li C, Peng Y, et al. Small-molecule BH3 mimetic and pan-Bcl-2 inhibitor AT-101 enhances the antitumor efficacy of cisplatin through inhibition of APE1 repair and redox activity in non-small-cell lung cancer. Drug Des Devel Ther. 2015;9:2887-910.

13. Hirayama S, Ishii G, Nagai K, Ono S, Kojima M, Yamauchi C, Aokage K, Hishida T, Yoshida J, Suzuki K, et al. Prognostic impact of CD204-positive macrophages in lung squamous cell carcinoma: possible contribution of Cd204-positive macrophages to the tumor-promoting microenvironment. Journal of thoracic oncology: official publication of the International Association for the Study of Lung Cancer. 2012;7(12):1790-7.

14. Ni L, Feng Y, Wan H, Ma Q, Fan L, Qian Y, Li Q, Xiang Y, Gao B. Angiotensin-(1-7) inhibits the migration and invasion of A549 human lung adenocarcinoma cells through inactivation of the PI3K/Akt and MAPK signaling pathways. Oncol Rep. 2012;27(3):783-90.

15. Wu HH, Chu YC, Wang L, Tsai LH, Lee MC, Chen CY, Shieh SH, Cheng YW, Lee H. Cytoplasmic Ape1 expression elevated by p53 aberration may predict survival and relapse in resected non-small cell lung cancer. Ann Surg Oncol. 2013;20(Suppl 3):336-47.

16. Manent J, Banerjee S, de Matos Simoes R, Zoranovic T, Mitsiades C, Penninger JM, Simpson KJ, Humbert PO, Richardson HE. Autophagy suppresses Ras-driven epithelial tumourigenesis by limiting the accumulation of reactive oxygen species. Oncogene. 2017;36(40):5658-60.

17. Schmukler E, Kloog Y, Pinkas-Kramarski R. Ras and autophagy in cancer development and therapy. Oncotarget. 2014;5(3):577-86.

18. Qian C, Li M, Sui J, Ren T, Li Z, Zhang L, Zhou L, Cheng Y, Wang D: Identification of a novel potential antitumor activity of gossypol as an APE1/Ref-1 inhibitor. Drug design, development and therapy 2014, 8:485-496.

19. Hancock CN, Stockwin LH, Han B, Divelbiss RD, Jun JH, Malhotra SV, Hollingshead MG, Newton DL. A copper chelate of thiosemicarbazone NSC 689534 induces oxidative/ER stress and inhibits tumor growth in vitro and in vivo. Free Radic Biol Med. 2011;50(1):110-21.

20. Ma NX, Sun W, Wu J, Liu SL, Weng L, Liu YQ, Pu WX, Wu TT, Ding XL, Huang NG, et al. Compound Wumei Powder Inhibits the Invasion and Metastasis of Gastric Cancer via Cox-2/PGE2PI3K/AKT/GSK3beta/beta-Catenin Signaling Pathway. Evidence-based complementary alternative medicine: eCAM. 2017;2017:3039450.

21. Liu B, Yan S, Qu L, Zhu J. Celecoxib enhances anticancer effect of cisplatin and induces anoikis in osteosarcoma via PI3K/Akt pathway. Cancer cell international. 2017;17:1.

22. Tasca KI, Correa CR, Caleffi JT, Mendes MB, Gatto M, Manfio VM, de Camargo CC, Tavares FC, Biasin M, de Souza LDR. Asymptomatic HIV People Present Different Profiles of sCD14, sRAGE, DNA 
Damage, and Vitamins, according to the Use of CART and CD4(+) T Cell Restoration. Journal of immunology research. 2018;2018:7531718.

23. Vascotto C, Bisetto E, Li M, Zeef LA, D'Ambrosio C, Domenis R, Comelli M, Delneri D, Scaloni A, Altieri $F$, et al: Knock-in reconstitution studies reveal an unexpected role of Cys-65 in regulating APE1/Ref-1 subcellular trafficking and function. Molecular biology of the cell 2011, 22(20):3887-3901.

\section{Figures}

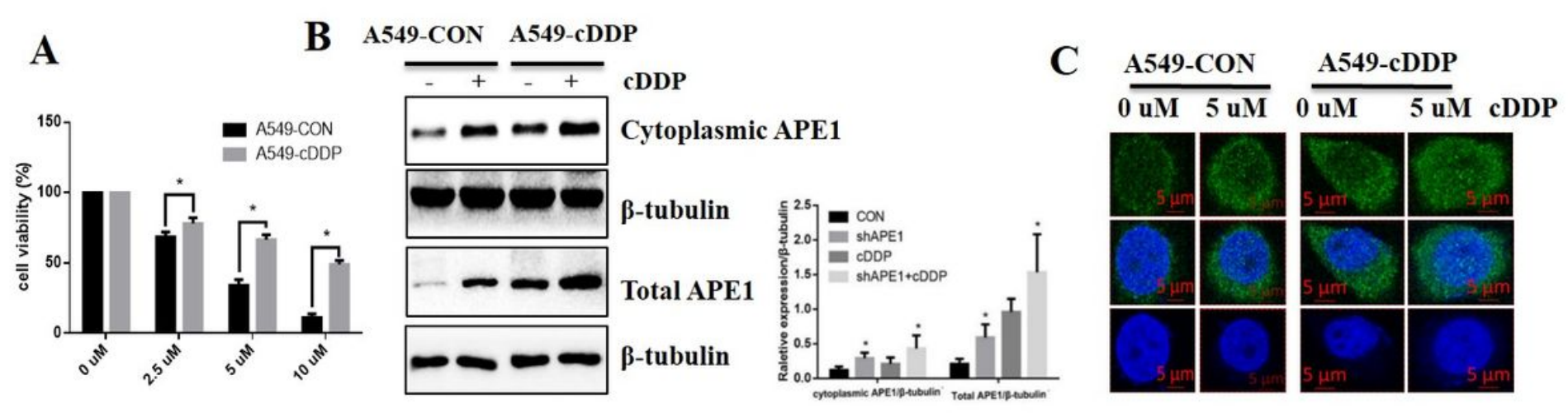

D

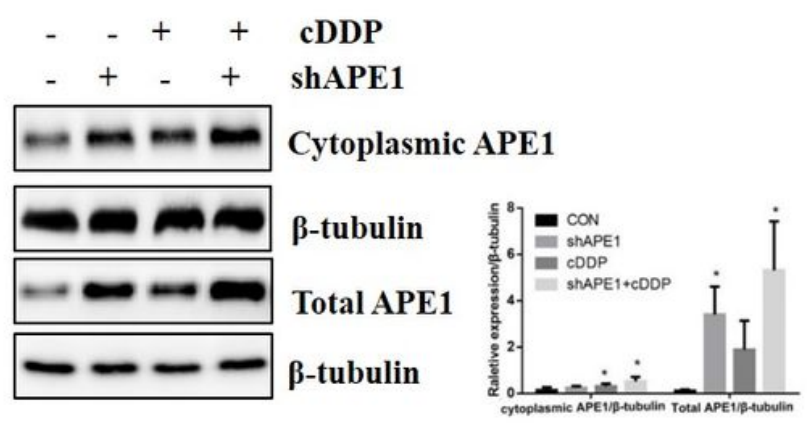

$\mathbf{E}$

$\mathbf{F}$

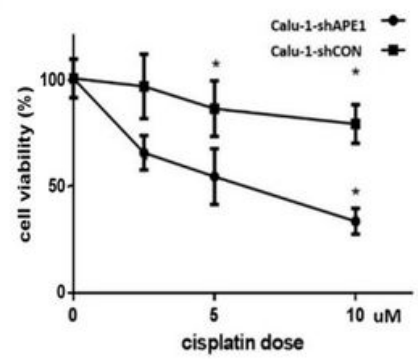

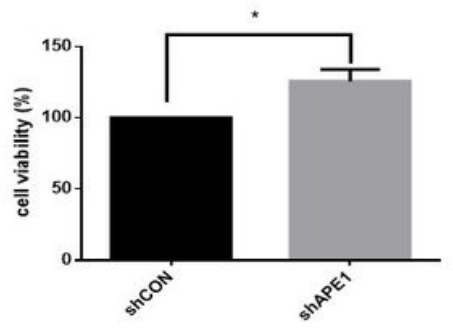

Figure 1

Overexpression of cytoplasmic APE1 induces cisplatin resistance. (A) A549 and A549/cDDP cells were seeded into 96 -well plates and treated with different concentrations of cisplatin (CDDP) for $24 \mathrm{~h}$. Cell proliferation was determined by the CCK-8 method. The columns represent the means \pm SD of three independent experiments, ${ }^{*} P<0.05$ versus the vehicle group, ${ }^{\star} \times P<0.001$ versus the vehicle group. (B) A549CON and A549-cDDP cells were either treated with $5 \mu \mathrm{M} \mathrm{cDDP}$ for $24 \mathrm{~h}$ or left untreated. Protein expression was determined by western blot assay. The columns represent the means \pm SD of three independent experiments, ${ }^{*} \mathrm{P}<0.05$ versus the vehicle group, ${ }^{* *} \mathrm{P}<0.001$ versus the vehicle group. The columns represent the means \pm SD of three independent experiments. (C) Immunofluorescence assay shows the accumulation of APE1 in A549 cells. (D) Calu-1 cells transfected with APE1-NLS mutation plasmids. Calu-1-shCON and Calu-1-shAPE1 cells were either treated with $5 \mu \mathrm{M}$ cisplatin for $24 \mathrm{~h}$ or left untreated. The columns represent the means \pm SD of three independent experiments, ${ }^{*} P<0.05$ versus the vehicle group, ${ }^{*} \mathrm{P}<0.001$ versus the vehicle group. $(\mathrm{E}-\mathrm{F})$ Calu-1-shCON and Calu-1-shAPE1 were seeded 
into 96-well plates and treated with different concentrations of cisplatin (cDDP) for $24 \mathrm{~h}$. Cell proliferation was determined. The columns represent the means \pm SD of three independent experiments, *P<0.05 versus the vehicle group, ${ }^{*} \mathrm{P}<0.001$ versus the vehicle group.

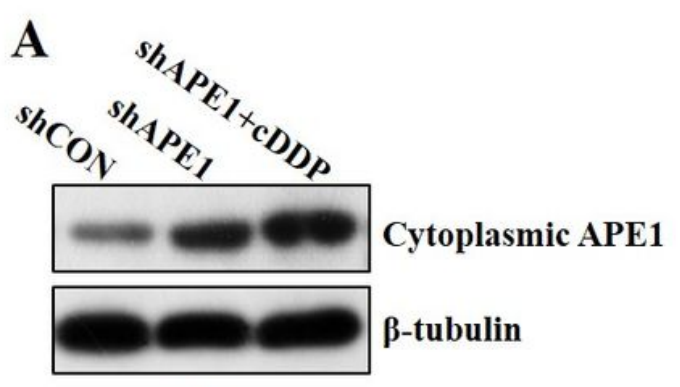

B
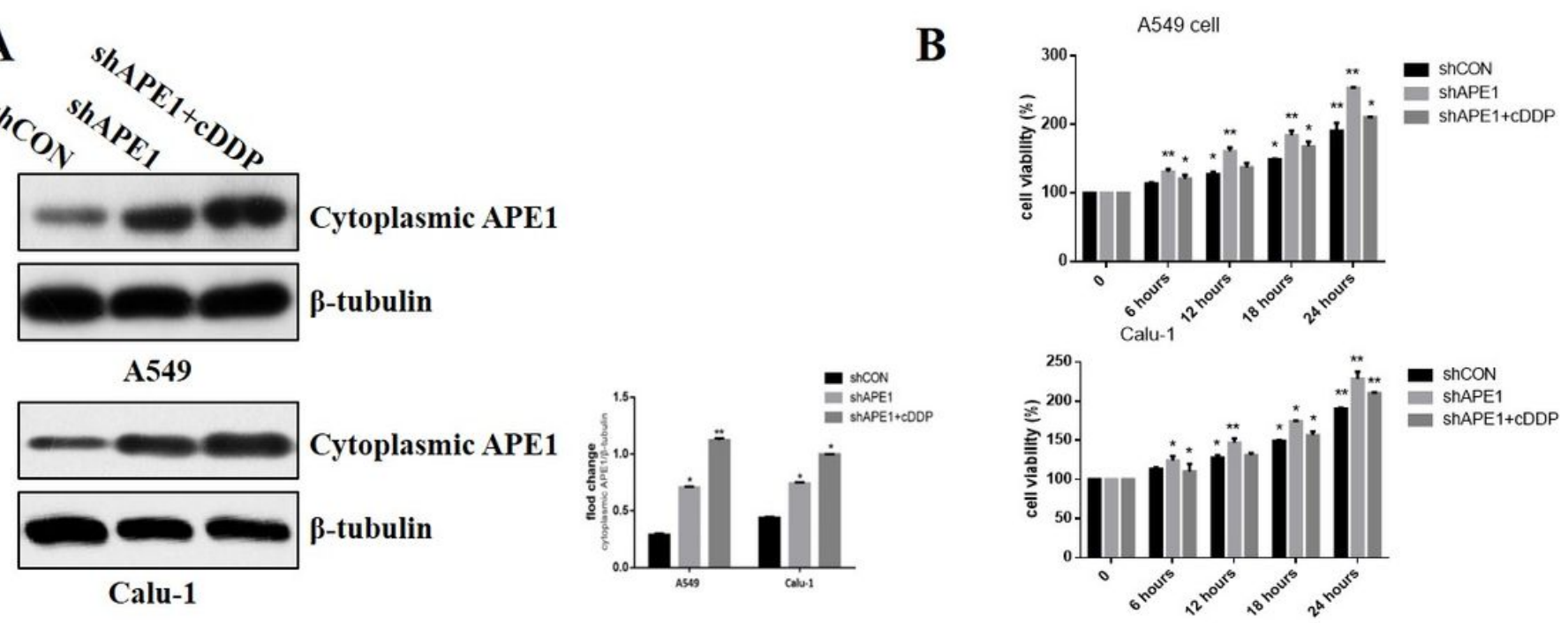

C

D

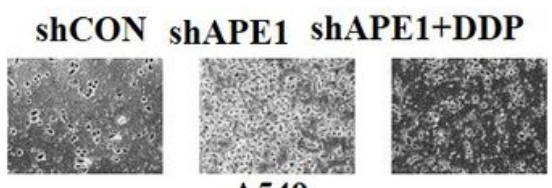

shCON shAPE1 shAPE1+DDP shCON shAPE1 shAPE1+DDP
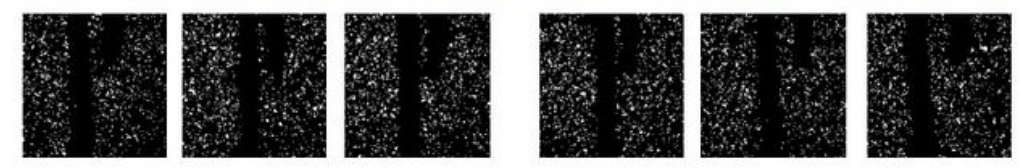

0h

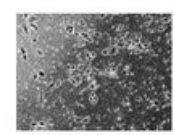

A549
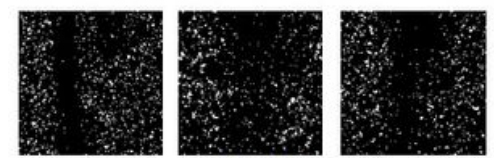

A549
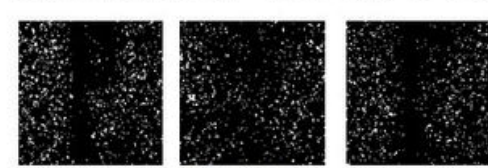

Callu-1
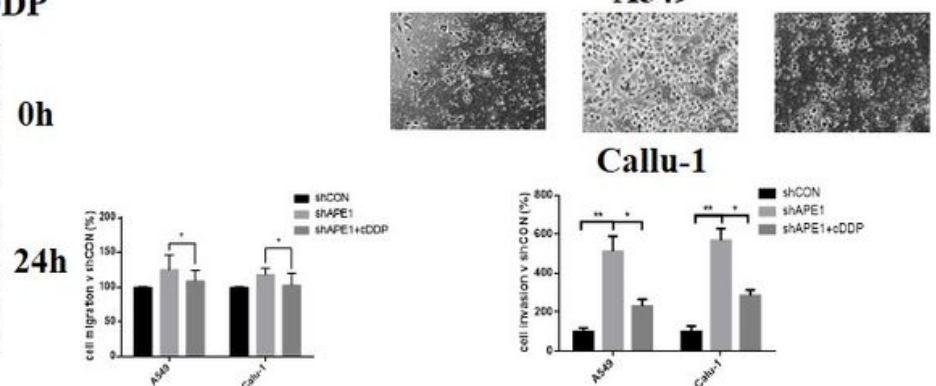

Callu-1

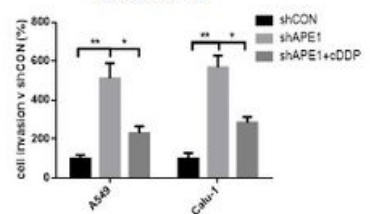

Figure 2

Effects of cytoplasmic APE1 in lung cancer cell growth, migration and invasion in vitro. (A) A549 cells transfected with APE1-NLS mutation plasmids. A549-shCON and A549-shAPE1; Calu-1-shCON and Calu1-shAPE1 cells were either treated with $5 \mu \mathrm{M}$ cisplatin (CDDP) for $24 \mathrm{~h}$ or left untreated. The protein expression level was determined by western blot. The columns represent the means \pm SD of three independent experiments, ${ }^{*} \mathrm{P}<0.05$ versus the vehicle group, ${ }^{*} \mathrm{P}<0.001$ versus the vehicle group. (B) A549shCON and A549-shAPE1; Calu-1-shCON and Calu-1-shAPE1 cells were seeded into 96-well plates and treated with $5 \mu \mathrm{M}$ cisplatin for different times. Cell proliferation was determined by CCK-8 assay. The columns represent the means \pm SD of three independent experiments, ${ }^{*} \mathrm{P}<0.05$ versus the vehicle group, ${ }^{*} \mathrm{P}<0.001$ versus the vehicle group. (C) Migratory ability of A549-shCON and A549-shAPE1; Calu-1shCON and Calu-1-shAPE1 cells treated with $5 \mu \mathrm{M}$ cisplatin for $24 \mathrm{~h}$ or left untreated was tested using a wound-healing assay. The gaps in the indicated groups were imaged at 0 and $24 \mathrm{~h}$; the relative migration distances of three independent experiments are shown. The columns represent the means \pm SD of three independent experiments, ${ }^{*} \mathrm{P}<0.05$ versus the vehicle group, $* * P<0.001$ versus the vehicle group. (D) The 
invasion capability of A549-shCON and A549-shAPE1; Calu-1-shCON and Calu-1-shAPE1 cells treated with $5 \mu \mathrm{M}$ cisplatin for $48 \mathrm{~h}$ or left untreated were determined using Transwell assays. The columns represent the means $\pm S D$ of three independent experiments, ${ }^{*} P<0.05$ versus the vehicle group, ${ }^{* *} P<0.001$ versus the vehicle group.

A

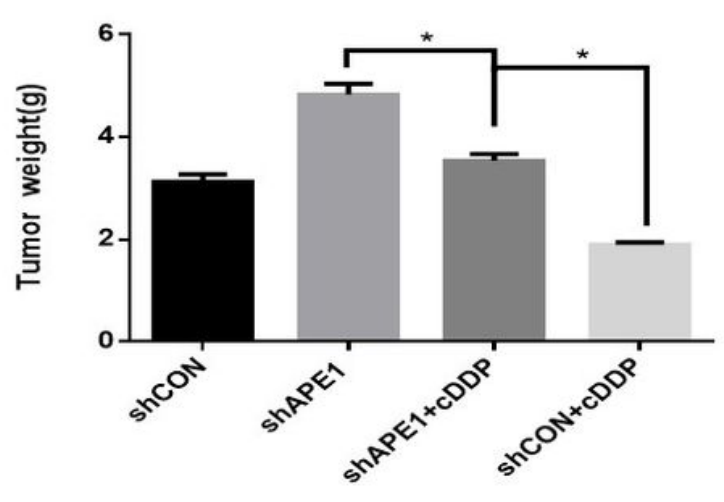

C
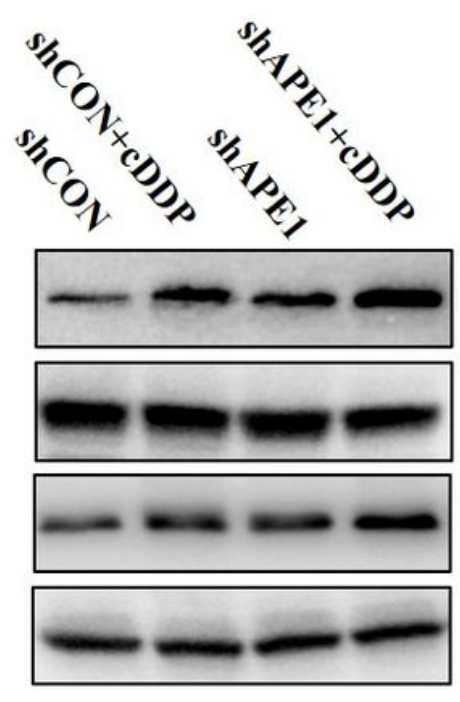

Cytoplasmic APE1

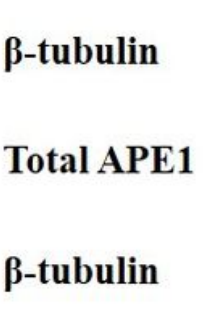

B

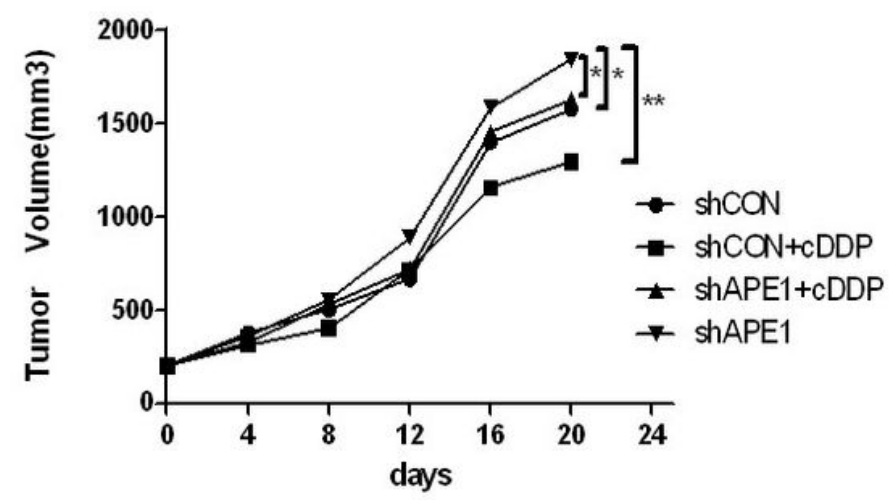

D

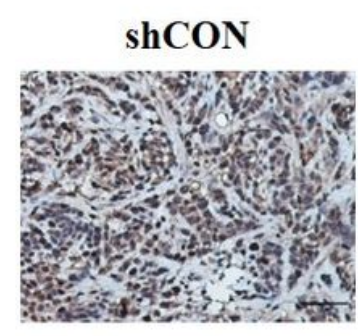
shCON+cDDP

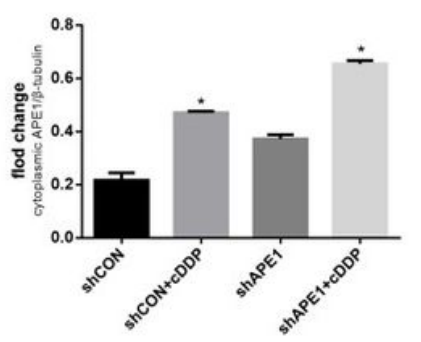

ShAPE1

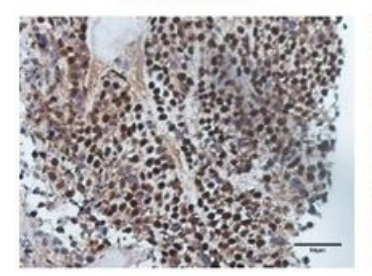

ShAPE1+cDDP

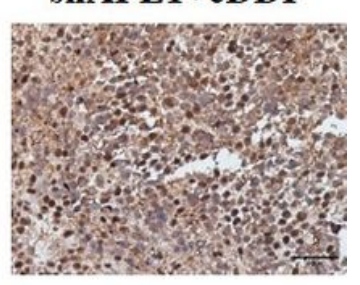

APE1

Figure 3

Overexpression of cytoplasmic APE1 promotes tumor growth in vivo. Nude mice were injected with A549 cells stably transfected with a plasmid containing NLS-mutated APE1. Xenograft models were divided into three groups (10 mice/group) and treated for 14 days with cisplatin $4 \mathrm{mg} / \mathrm{kg}$ once daily by intraperitoneal injection. Tumor weight was measured on day $14(\mathrm{~A})$, The columns represent the means \pm SD of three independent experiments, ${ }^{*} P<0.05$ versus the vehicle group, ${ }^{*} P<0.001$ versus the vehicle group. Tumor volume was measured using a caliper (B). Tumor tissues isolated from xenografts after cisplatin treatment were subjected to western blot analysis (C). The columns represent the means \pm SD of three independent experiments, ${ }^{*} P<0.05$ versus the vehicle group, ${ }^{*} P<0.001$ versus the vehicle group, and immunohistochemistry analysis of APE1 (D). 

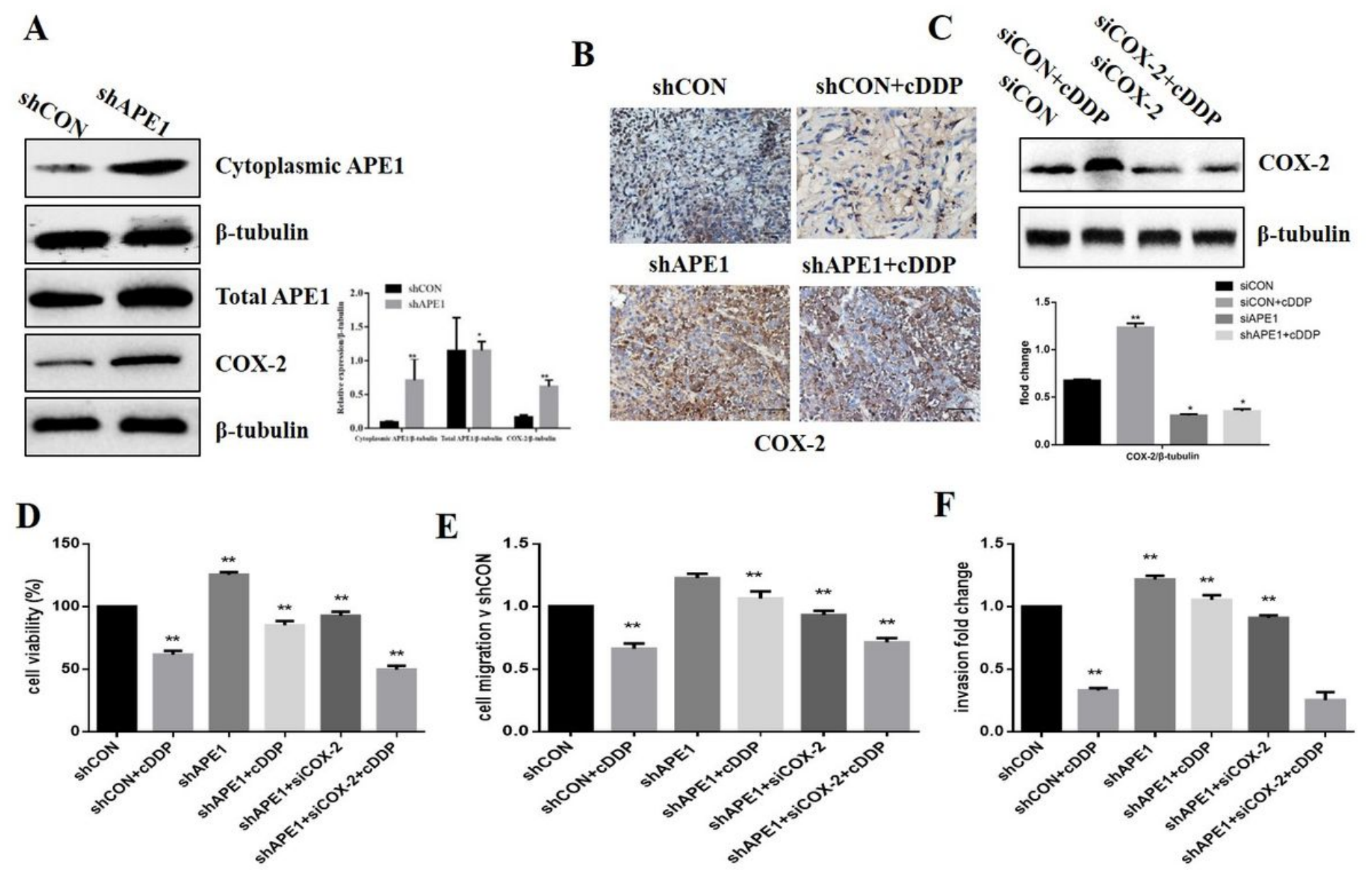

Figure 4

The effect of cytoplasmic APE1 on lung cancer cells was affected by COX-2 levels. (A) A549 cells transfected with APE1-NLS mutation plasmids. Overexpression of cytoplasmic APE1 increased COX-2 expression. The columns represent the means \pm SD of three independent experiments. The columns represent the means $\pm S D$ of three independent experiments, ${ }^{*} P<0.05$ versus the vehicle group, ${ }^{*} \mathrm{P}<0.001$ versus the vehicle group. (B) Tumor tissues isolated from xenografts after cisplatin treatment were subjected to immunohistochemistry analysis, and increased cytoplasmic APE1 expression was consistent in vivo. (C) A549 cells transfected with COX-2 mutation plasmids. siCON and siCOX-2 cells were either treated with $5 \mu \mathrm{M}$ cisplatin (cDDP) for $24 \mathrm{~h}$ or left untreated. The protein expression level was determined by western blot. The columns represent the means \pm SD of three independent experiments, ${ }^{*} P<0.05$ versus the vehicle group, ${ }^{*} P<0.001$ versus the vehicle group. (D) A549-shCON, A549-shAPE1 and A549-shAPE1+siCOX-2 cells were either treated with $5 \mu \mathrm{M}$ cisplatin for $24 \mathrm{~h}$ or left untreated. Cell proliferation was determined. The columns represent the means \pm SD of three independent experiments, ${ }^{*} P<0.05$ versus the vehicle group, ${ }^{*} P<0.001$ versus the vehicle group. (E) Migratory ability of cell was tested using wound-healing assay. The gaps of indicated groups were imaged at 0 and $24 \mathrm{~h}$; relative migration distance of three independent experiments. The columns represent the means \pm SD of three independent experiments, ${ }^{*} P<0.05$ versus the vehicle group, ${ }^{*} P<0.001$ versus the vehicle group. $(F)$ The invasion capability of cells treated with $5 \mu \mathrm{M}$ cisplatin for $48 \mathrm{~h}$ or left untreated was determined using 
Transwell assays. The columns represent the means \pm SD of three independent experiments, ${ }^{*} P<0.05$ versus the vehicle group, ${ }^{* *} \mathrm{P}<0.001$ versus the vehicle group.

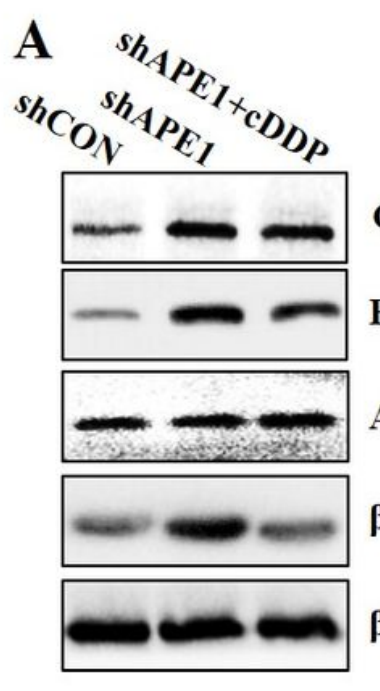

C

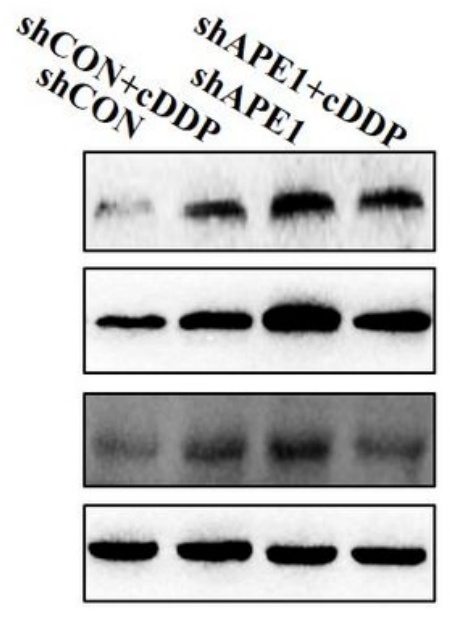

$\operatorname{COX}-2$

P-Akt

Akt

$\beta$-catenin

$\beta$-tubulin
B
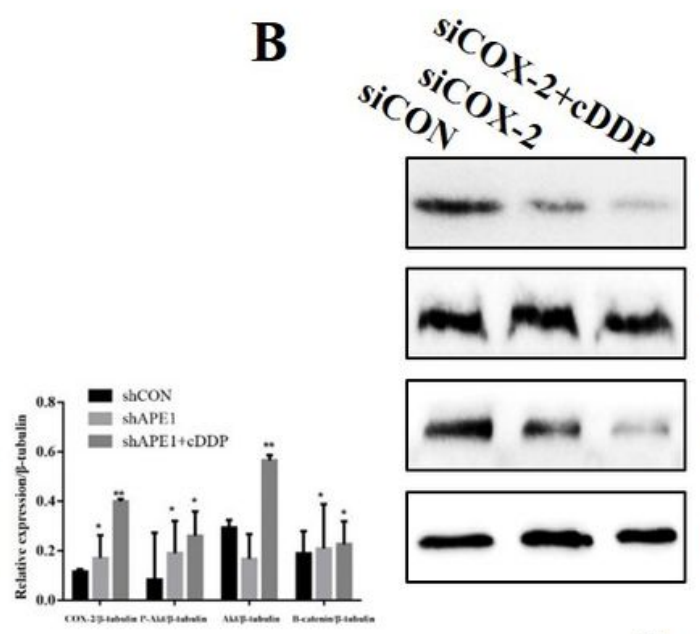

P-Akt

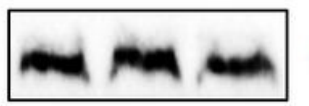

Akt
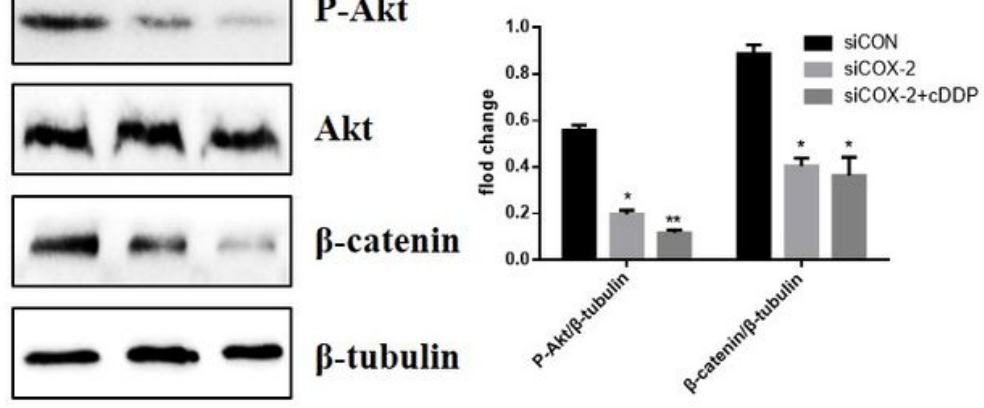

$\mathbf{E}$

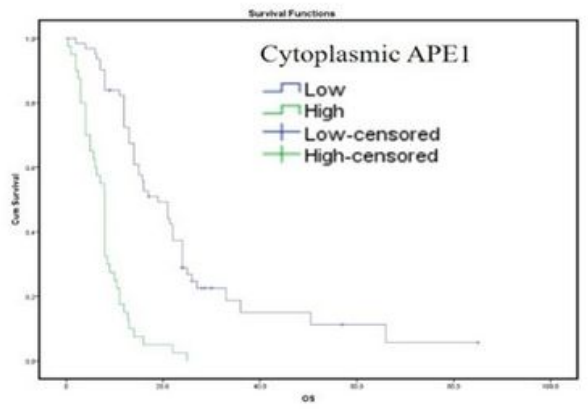

COX-2

P-Akt

$\beta$-catenin

$\beta$-tubulin
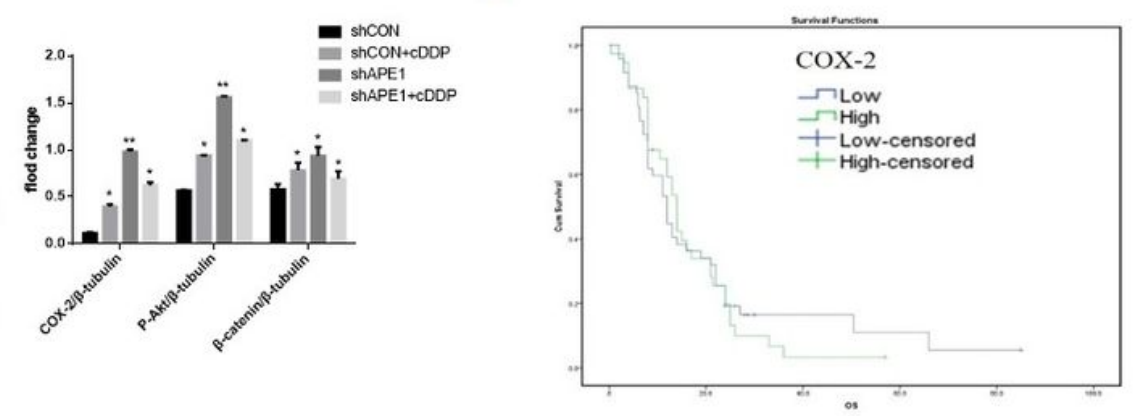

Figure 5

Cytoplasmic APE1 inhibits the Akt/ $\beta$-catenin signaling pathway by targeting COX-2 in lung cancer cells. (A) A549 cells transfected with APE1-NLS mutation plasmids. A549-shCON and A549-shAPE1 cells were either treated with $5 \mu \mathrm{M}$ cisplatin for $24 \mathrm{~h}$ or left untreated. The protein expression level was determined by western blot. The columns represent the means $\pm S D$ of three independent experiments, ${ }^{*} P<0.05$ versus the vehicle group, ${ }^{*} P<0.001$ versus the vehicle group. (B) A549 cells transfected with COX-2 knockdown plasmids. A549-siCON and A549-siAPE1 cells were either treated with $5 \mu \mathrm{M}$ cisplatin for $24 \mathrm{~h}$ or left untreated. The protein expression level was determined by western blot, and knockdown of COX-2 inhibited P-Akt and $\beta$-catenin in A549 cells. The columns represent the means \pm SD of three independent experiments, ${ }^{*} P<0.05$ versus the vehicle group, ${ }^{*} P<0.001$ versus the vehicle group. (C) Tumor tissues isolated from xenografts after cisplatin treatment were subjected to western blot analysis in vivo, and overexpression of cytoplasmic APE1 increased P-Akt and $\beta$-catenin in vivo. The columns represent the 
means $\pm S D$ of three independent experiments, ${ }^{*} P<0.05$ versus the vehicle group, ${ }^{\star *} P<0.001$ versus the vehicle group. Kaplan-Meier analysis comparing the OS between patients with high and low expression of APE1 and COX-2. Prognostic significance of APE1 and COX-2 in 124 lung cancer patients was assessed by Kaplan-Meier analyses: patients with low levels of cytoplasmic APE1 had better OS (D). Patients with higher COX-2 levels had poorer OS (E). The P-values were obtained by the log-rank test.* $P<0.05$; $P<$ 0.01 , vs. control.

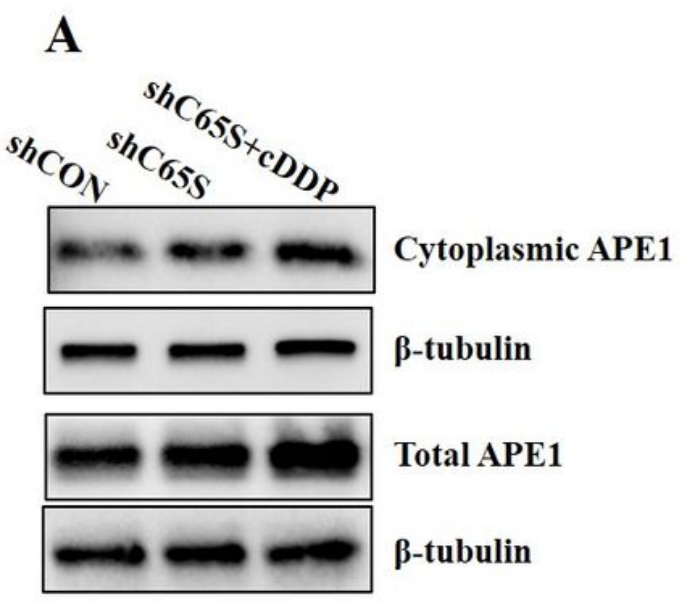

B

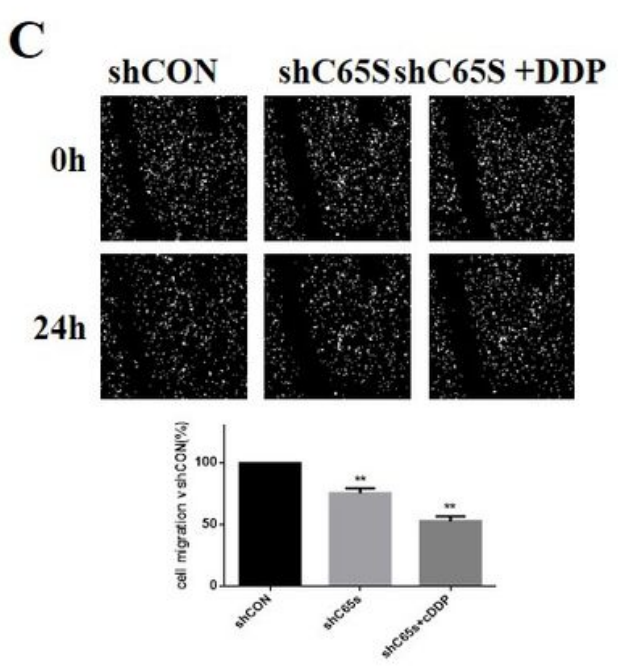

D
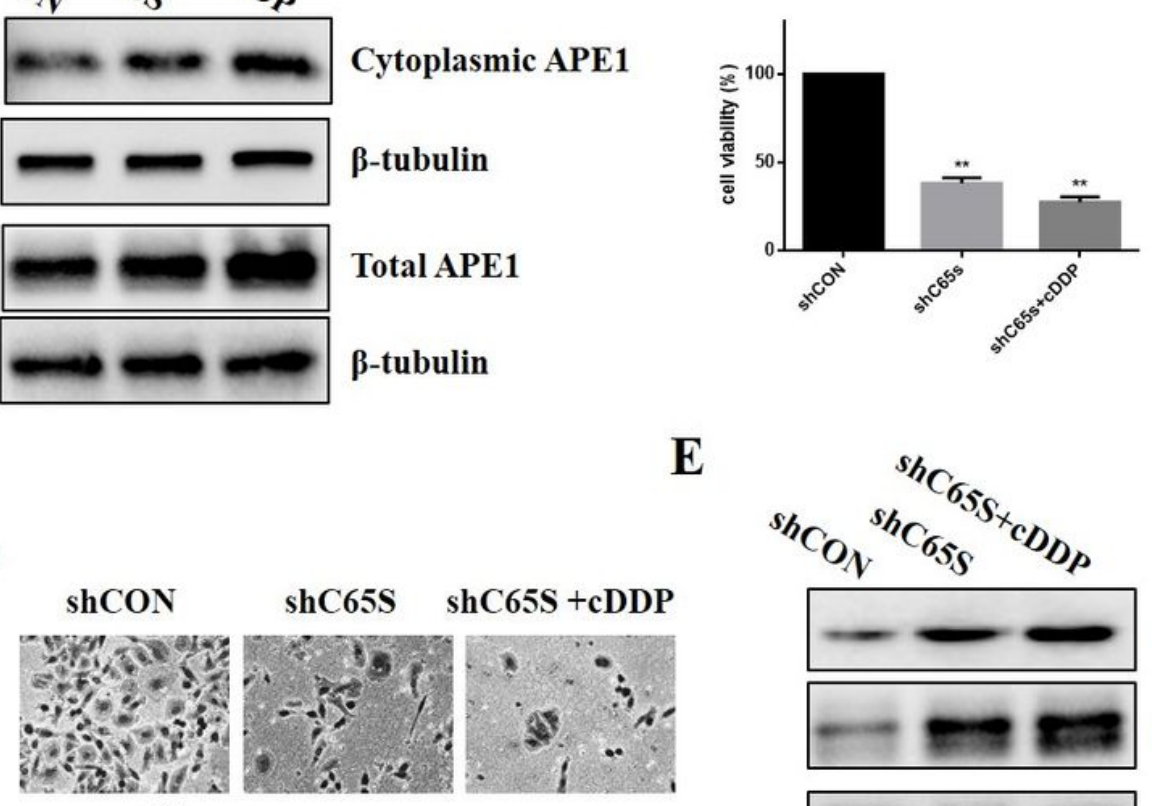

E
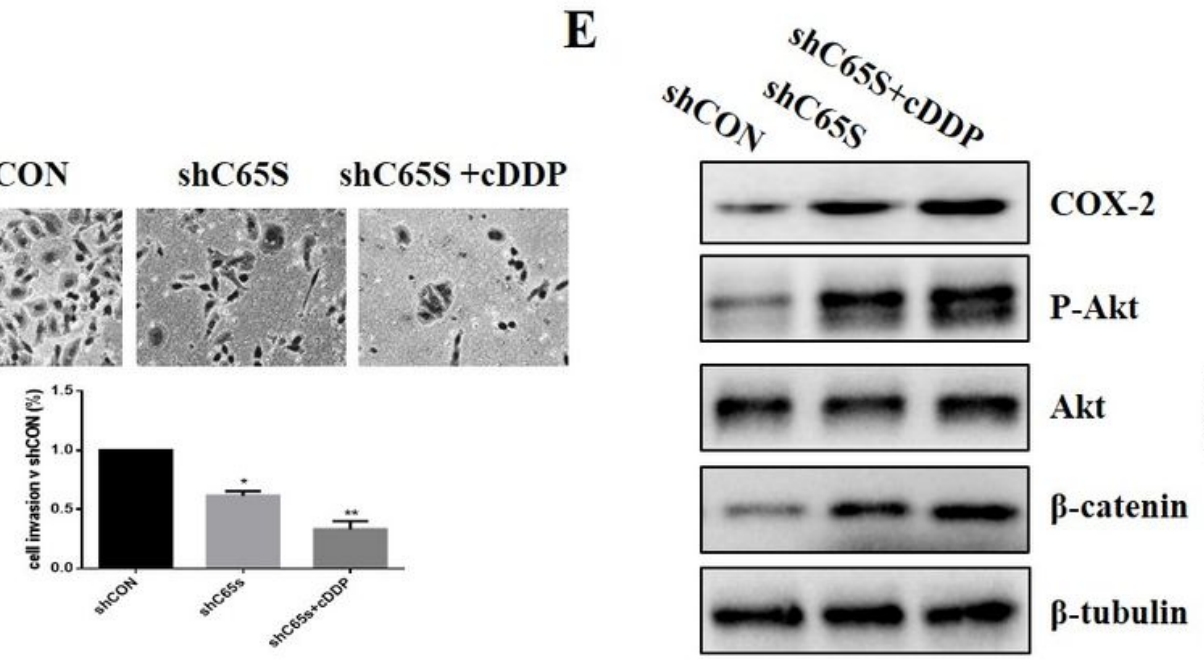

\section{Figure 6}

Cytoplasmic APE1 is downregulated by mutation of the APE1-C65 site. (A) A549 cells transfected with APE1-C65 site mutation plasmids. A549-shCON and A549-shC65s cells were either treated with $5 \mu \mathrm{M}$ cisplatin for $24 \mathrm{~h}$ or left untreated. The protein expression level was determined by western blot. (B) A549 cells transfected with APE1-C65 site mutation plasmids. A549-shCON and A549-shC65s cells were either treated with $5 \mu \mathrm{M}$ cisplatin for $24 \mathrm{~h}$ or left untreated. Cell proliferation was determined by CCK-8 assay. The columns represent the means \pm SD of three independent experiments, ${ }^{*} P<0.05$ versus the vehicle group, $* \star P<0.001$ versus the vehicle group. (C) Migratory ability of cells was tested using a wound-healing assay. The gaps of indicated groups were imaged at 0 and $24 \mathrm{~h}$; The columns represent the means \pm SD of three independent experiments, ${ }^{*} P<0.05$ versus the vehicle group, ${ }^{*} P<0.001$ versus the vehicle group. (D) A549 cells transfected with APE1-C65 site mutation plasmids. A549-shCON and A549-shC65s cells 
were either treated with $5 \mu \mathrm{M}$ cisplatin for $48 \mathrm{~h}$ or left untreated. The invasion capability of cell was determined using Transwell assays. The columns represent the means \pm SD of three independent experiments, ${ }^{*} P<0.05$ versus the vehicle group, ${ }^{*} \mathrm{P}<0.001$ versus the vehicle group. (E) A549 cells transfected with APE1-C65 site mutation plasmids. A549-shCON and A549-shC65s cells were either treated with $5 \mu \mathrm{M}$ cisplatin for $24 \mathrm{~h}$ or left untreated. The overexpression of APE1 with mutation at the C65 site significantly increased the expression of COX-2, P-Akt and $\beta$-catenin after cisplatin treatment in A549 cells. The columns represent the means \pm SD of three independent experiments, $* P<0.05$ versus the vehicle group, ${ }^{\star *} \mathrm{P}<0.001$ versus the vehicle group.

\section{Supplementary Files}

This is a list of supplementary files associated with this preprint. Click to download.

- renamedc31f3.docx

- renamedc31f3.docx 